\title{
(6) OPEN ACCESS \\ Loss of histone variant macroH2A2 expression associates with progression of anal neoplasm
}

\author{
Wan-Hsiang Hu, ${ }^{1,2,3}$ Katsumi Miyai, ${ }^{4}$ Judith C Sporn, ${ }^{5}$ Linda Luo, ${ }^{1,2}$ \\ Jean Y J Wang, ${ }^{2,6}$ Bard Cosman, ${ }^{1,7}$ Sonia Ramamoorthy ${ }^{1,2}$
}

For numbered affiliations see end of article.

\section{Correspondence to} Dr Sonia Ramamoorthy, Department of Surgery, Rebecca and John Moores Cancer Center, University of California San Diego Health System, 3855 Health Sciences Drive, La Jolla CA 92093, USA sramamoorthy@mail.ucsd.edu

Received 27 August 2015 Revised 16 November 2015 Accepted 20 November 2015 Published Online First 10 December 2015

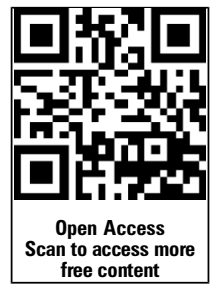

CrossMark

To cite: Hu W-H, Miyai K, Sporn JC, et al. J Clin Pathol 2016:69:627-631

\section{ABSTRACT}

Aims The macroH2A histone variants are epigenetic marks for inactivated chromatin. In this study, we examined the expression of macroH2A2 in anal neoplasm from anal intraepithelial neoplasia (AIN) to anal squamous cell carcinoma (SCC).

Methods AIN and anal SCC samples were analysed for macroH2A2 expression, HIV and human papilloma virus (HPV). The association of macroH2A2 expression with clinical grade, disease recurrence, overall survival and viral involvement was determined.

Results macroH2A2 was expressed in normal squamous tissue and lower grade AIN (I and II). Expression was lost in $38 \%$ of high-grade AIN (III) and $71 \%$ of anal SCC ( $p=0.002)$. Patients with AIN with macroH2A2-negative lesions showed earlier recurrence than those with macroH2A2-positive neoplasm $(p=0.017)$. With anal $S C C$, macroH2A2 loss was more prevalent in the HPV-negative tumours.

Conclusions Loss of histone variant macroH2A2 expression is associated with the progression of anal neoplasm and can be used as a prognostic biomarker for high-grade AIN and SCC.

\section{INTRODUCTION}

Among the core histones, the H2A family has the highest number of variants, including $\mathrm{H} 2 \mathrm{AX}$, $\mathrm{H} 2 \mathrm{AZ}$ and macroH2A. ${ }^{1}$ The macroH2A histone variants are conserved in vertebrates and are encoded by two genes: H2AFY for macroH2A1 and H2AFY2 for macroH2A2. Each of these macrohistone variants contain an $\mathrm{N}$-terminal H2A-histone domain and a C-terminal Macro domain ${ }^{2}{ }^{3}$ that is found in other non-histone proteins, for example, poly-ADP ribose polymerases (PARP-9, -14 and -15) and poly-ADP ribose glycohydrolase. ${ }^{4}$ The Macro domain of mH2A1.1 binds to ADP ribose that is generated from NAD by enzymes such as the NAD-dependent histone deacetylase SIRT1. ${ }^{4-6}$ The ligand for the Macro domain of macroH2A2 is presently unknown. The macroH2A histones are enriched on the inactivated $\mathrm{X}$-chromosome but not required for the establishment of X-inactivation. ${ }^{7-9}$ The macroH2A histones are also epigenetic and repressive markers of many developmental regulator genes in the male pluripotent cells. ${ }^{10}$ MacroH2A variants have been shown to act cooperatively as a barrier to the reprogramming of somatic cells with macroH2A2 being the predominant inhibitor. ${ }^{11}$ In mice, the combined knockout of macroH2A1 and macroH2A2 did not affect early development but affected the growth and the fertility of adult mice. ${ }^{12}$ In embryonic stem (ES) cells, macroH2A-containing nucleosomes are found at promoters and in intergenic regions in ES cells. ${ }^{13}$ The dynamic replacement of nucleosomes with macroH2A2 in ES cells was dampened, whereas the stable association of macroH2A2 nucleosomes with intergenic regions was expanded upon differentiation. ${ }^{13}$ It was found that macroH2A2 is required for EPO-induced erythroid maturation. ${ }^{14}$

Previous studies have also suggested that macroH2A histones have tumour suppressor function. ${ }^{15} 16$ The Ladurner group was the first to report that the expression of macroH2A1 and macroH2A2 inversely correlated with proliferation in human breast and lung cancer. They also showed that the levels of macroH2A expression could be used as predictive biomarkers for lung cancer recurrence. ${ }^{17}$ In a study of melanoma, the expression of macroH2A2 was found in the benign naevi and during radial growth, but lost in the vertical growth phase and in metastatic melanoma. ${ }^{18}$ The expression of macroH $2 \mathrm{~A}$ histones at the protein level in other cancer types has not been investigated. Examination of The Cancer Genome Atlas (TCGA) database for H2AFY and H2AFY2 sequence, copy number and RNA levels showed alterations in only a small fraction of cases $(<10 \%)$ across TCGA cancer types. Analyses of the RNA levels found that significant reduction in H2AFY2, but not H2AFY, RNA expression (outliners) occurred in a small fraction of cases among several cancer types. Therefore, it appears that macroH2A2 was more likely to be reduced than macroH2A1 in human tumour samples. However, the TCGA network has not examined the expression of macroH $2 \mathrm{~A}$ histone proteins.

In this study, we investigated the expression of macroH2A2 protein in anal dysplasia and anal squamous cell carcinoma (SCC). In the USA, neoplasm of the anal squamous tissue has been increasing steadily in recent years, with estimated new cases and deaths of 7210 and 950, respectively, in $2014 .{ }^{19}$ SCC is likely to progress from a continuum of precancerous lesions known as anal intraepithelial neoplasia (AIN). These precancerous AIN lesions are divided into three categories according to degrees of histological atypia. ${ }^{20}$ In another classification, AIN I is known as low-grade anal squamous intraepithelial lesion, and AIN II and III are combined into the group of high-grade anal squamous intraepithelial lesion (HSIL). ${ }^{20}{ }^{21}$ Infections with human papilloma virus (HPV) and HIV are important risk factors for anal SCC. ${ }^{22}$ Their individual effect on the development of anal SCC is difficult to assess due to the prevalence of HPV and a higher risk of contracting HPV in patients who 
are HIV positive. ${ }^{23}$ In this study, we examined the expression of macroH2A2 by immunohistochemistry staining of AIN and anal SCC samples in association with the status of HIV and HPV infections.

\section{MATERIALS AND METHODS}

Patients and specimens

Tissue samples including AIN and anal SCC were collected from the patients in UC San Diego Medical Center, California, between 2000 and 2008. Patients' clinical characteristics were also summarised. In patients with AIN, treatment included excision and ablation. Time to recurrence was calculated between onset and first recurrence after treatment. According to their clinical stage, patients with anal SCC received the treatments including local excision, concurrent chemoradiotherapy or abdominoperineal resection. The dose of radiotherapy typically ranged from 5040 to $5940 \mathrm{cGy}$ in 28 fractions. The regimens of chemotherapy were 5-fluorouracil with mitomycin or cisplatin. Patients with anal SCC who were progressive after the day of tissue biopsy were excluded from recurrence evaluation. We evaluated the 5-year recurrence-free survival of AIN and 5-year disease-free survival of patients with anal SCC according to the interval of survival without AIN and anal SCC recurrence.

\section{Immunohistochemistry}

The sections cut from the formalin-fixed and paraffin-embedded tissues were deparaffinised in xylene and dehydrated in ethanol. They were then incubated in the Decloaking chamber (DC2002; Biocare Medical, Concord, California, USA) contain-

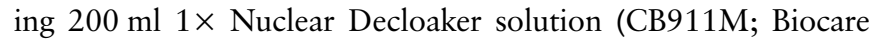
Medical). Endogenous peroxidase activity was blocked by incubation with Peroxidazed 1 (PX968; Biocare Medical). Background Sniper (BS966; Biocare Medical) was added for $1 \mathrm{~h}$ to block non-specific protein binding. The slides were incubated overnight at $4^{\circ} \mathrm{C}$ with macroH2A2 antibody ${ }^{17}$ and then processed using a MACH 4 Universal HRP-Polymer Kit (M4U534; Biocare Medical). Betazoid DAB (BDB2004; Biocare Medical) was used for chromogen development. The slides were counterstained with haematoxylin. The pathologist was responsible for reviewing the slides and unaware of patient's clinical data.

\section{HIV and HPV detection}

The data of HIV infection of patients with AIN and anal SCC were collected according to the report of UC San Diego Medical Center. In anal SCC, DNA tissue was extracted, purified and amplified from the samples. The individual HPV genotyping was detected by microarray method. The details were mentioned in the reference. ${ }^{24}$

\section{Statistical analysis}

Fisher's exact test was used to compare the difference of macroH2A2 loss fraction in AIN I, AIN II, AIN III and anal SCC samples. Kaplan-Meier curves of estimated time to recurrence and disease-free survival were generated and compared between the groups using a two-sided log-rank test. $p$ Value $<0.05$ was considered statistically significant. Statistical analysis was performed using Prism V.6 software and tools of in silico website.

\section{RESULTS}

macroH2A2 expression in AIN and anal SCC

A total of 41 patient samples, AIN I $(n=4)$, AIN II $(n=7)$, AIN III $(n=16)$ and anal SCC $(n=14)$, were examined in this study. Representative immunohistochemistry staining for macroH2A2 expression of positive $(\mathrm{P})$ and negative $(\mathrm{N})$ samples is shown in figure 1A. We separated the samples into four groups, AIN I, AIN II, AIN III and anal SCC, and determined the fraction of samples that were negative $(\mathrm{N})$ for macroH2A2 staining. As shown in figure $1 \mathrm{~B}$, macroH2A2 expression was found in $100 \%$ of AIN I and AIN II samples. However, macroH2A2 expression was lost in $38 \%$ of the AIN III and $71 \%$ of the anal SCC samples. These results show a statistically significant correlation between the loss of macroH2A2 expression and the progression of anal neoplasm from AIN I/II to AIN III and anal SCC $(p=0.002)$. The clinical-pathological characteristics of the patients with AIN and anal SCC and the status of macroH2A2 expression in this study are also summarised in table 1.

macroH2A2 expression and recurrence of AIN and anal SCC In this study, we determined the recurrence of lesions in patients with AIN over a 5-year follow-up period and we performed a Kaplan-Meier analysis of the time to first recurrence as a function of macroH2A2 expression. As shown in figure $2 \mathrm{~A}$, the time to recurrence was significantly shorter in the macroH2A2-negative group than the macroH2A2-positive group $(p=0.017)$. The relationship between disease-free survival of anal SCC patients and macroH2A 2 expression is illustrated in figure $2 \mathrm{~B}$.

Relationship of macroH2A2 loss with HPV and HIV infections With the anal SCC samples, we also determined the presence of HPV and HIV sequences in the genomic DNA and assessed the
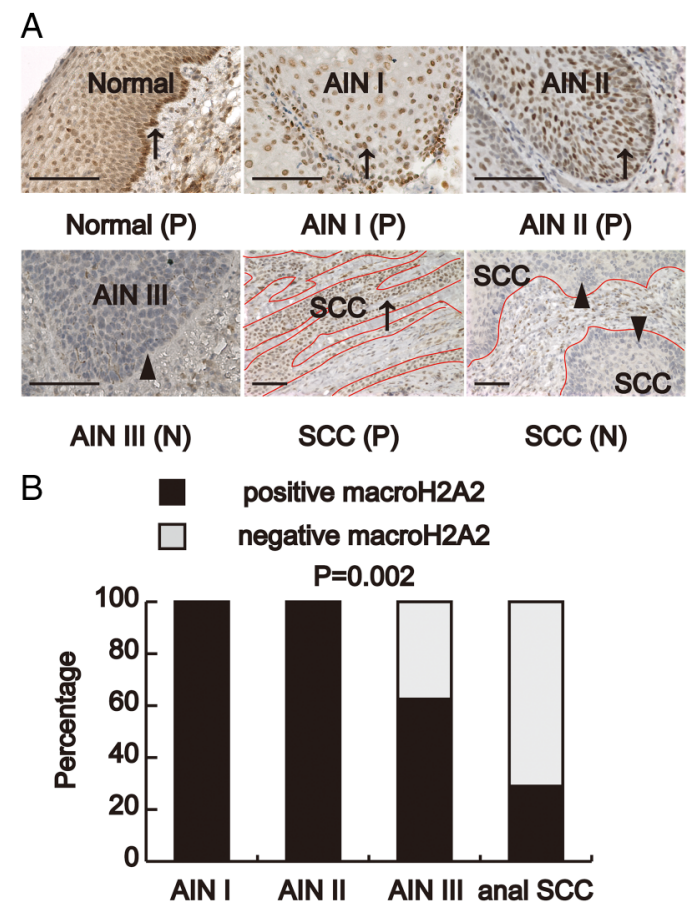

Figure 1 Immunohistochemistry stain for macroH2A2. (A) Representative samples of positive $(\mathrm{P})$ and negative $(\mathrm{N})$ macroH2A2 stain of normal anal squamous epithelial, anal intraepithelial neoplasm (AIN) and anal squamous cell carcinoma (SCC). Positive stain of macroH2A2 was brown colour in nucleus (arrow) and negative stain was blue haematoxylin counterstain (arrowhead). Anal SCC invading into adjuvant normal tissue was noted in the samples. (B) Loss of macroH2A2 expression associated with progression of anal neoplasm $(p=0.006$, Freeman-Halton extension of Fisher's exact test). Scale bar: $100 \mu \mathrm{m}$. 
Table 1 Clinicopathological characteristic of patients with AIN and anal SCC

\begin{tabular}{|c|c|}
\hline & Numbers \\
\hline AIN & 27 \\
\hline \multicolumn{2}{|l|}{ Age (years) } \\
\hline Median (range) & $44(24-69)$ \\
\hline \multicolumn{2}{|l|}{ Gender } \\
\hline Male & 23 \\
\hline \multicolumn{2}{|l|}{ Histology } \\
\hline I & 4 \\
\hline II & 7 \\
\hline III & 16 \\
\hline Positive macroH2A2 & 21 \\
\hline \multicolumn{2}{|l|}{ Recurrence (months) } \\
\hline 4 & 4 \\
\hline 12 & 12 \\
\hline Anal SCC & 14 \\
\hline \multicolumn{2}{|l|}{ Age (years) } \\
\hline Median (range) & $49(35-78)$ \\
\hline \multicolumn{2}{|l|}{ Gender } \\
\hline Male & 9 \\
\hline \multicolumn{2}{|l|}{ Differentiation } \\
\hline Well & 3 \\
\hline Moderate to poor & 11 \\
\hline Positive macroH2A2 & 4 \\
\hline \multicolumn{2}{|l|}{ Stage } \\
\hline I+II & 10 \\
\hline III+IV & 4 \\
\hline \multicolumn{2}{|l|}{ Treatment } \\
\hline Local excision & 5 \\
\hline CCRT & 6 \\
\hline APR & 2 \\
\hline Recurrence & 8 \\
\hline
\end{tabular}

AIN, anal intraepithelial neoplasia; CCRT, concurrent chemoradiotherapy; APR, abdominoperineal resection; SCC, squamous cell carcinoma.

relationship between macroH2A2 expression and viral infections. Among the 14 SCC samples, 8 were positive for HPV (of genotypes 16, 18, 33 and 43) and 7 were positive for HIV (table 2). Of the eight HPV-positive SCC samples, five were also positive for HIV. Of the six HPV-negative SCC samples, four were also negative for HIV. The viral involvements in these SCC samples were therefore consistent with co-infections with HIV and HPV. ${ }^{22}$

As shown in table 1 , only 4 of the 14 SCC samples were positive for macroH2A2 staining. Of those four samples, three were positive for HPV infection (table 2). As summarised in figure 3, the HPV-positive SCC samples were more likely to express macroH2A2. On the other hand, the HIV-positive SCC samples were less likely to express macroH2A2. With samples that were double positive or double negative for HPV and HIV, the probability of macroH2A expression was similar (figure 3).

\section{DISCUSSION}

The association between macroH2A2 protein expression and tumour progression has previously been investigated in lung cancer and melanoma. ${ }^{17} 18$ Before this study, the role of macroH2A2 in AIN and anal SCC was unknown. We show here that macroH2A2 expression is lost in the majority of anal SCC, and that the loss of this histone is also significantly associated with high-grade AIN and faster recurrence. This result supports
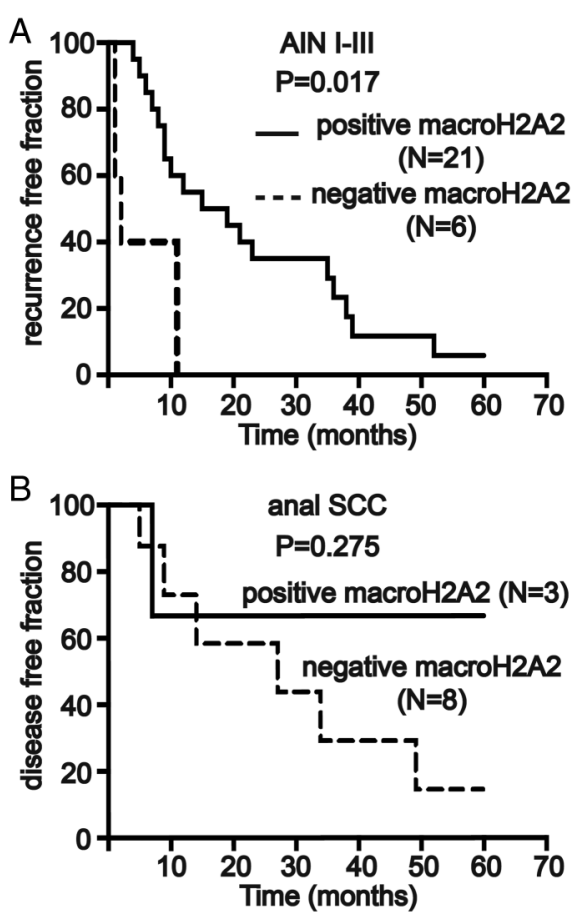

Figure 2 Association of macroH2A2 loss with anal intraepithelial neoplasia (AIN) recurrence. (A) Kaplan-Meier plots of time to recurrence among patients with AIN categorised by the status of macroH2A2 protein expression at the time of diagnosis. Loss of macroH2A2 expression in AIN is significantly associated with earlier recurrence $(p=0.017)$. (B) Kaplan-Meier plots of disease-free survival among patients with anal squamous cell carcinoma (SCC) categorised by the status of macroH2A2 protein expression. The loss of macroH2A2 expression is associated with reduced survival; however, due to limitation of sample size, this trend is not statistically significant.

the concept that macroH2A2 is likely to have a tumour suppression function in anal neoplastic development. Similar to cervical cancer evolving from premalignant lesions (cervical intraepithelial neoplasia, CIN), ${ }^{25}$ high-grade AIN, especially AIN III, is considered to be a precursor lesion to anal SCC. The malignant

Table 2 Summary of macroH2A2 expression, clinical stage and viral involvement in the anal SCC samples

\begin{tabular}{llll}
\hline macroH2A2 & Stage & HIV & HPV \\
\hline+ & I & - & 16 \\
+ & IV & - & - \\
+ & II & - & 16 \\
+ & I & + & 16 \\
- & II & + & 18 \\
- & IV & - & - \\
- & I & + & - \\
- & I & + & 33 \\
- & III & - & 16,33 \\
- & I & + & 16 \\
- & III & + & - \\
- & I & + & 16,43 \\
- & II & - & - \\
- & II & & - \\
\hline The HPV genotypes are indicated. &
\end{tabular}


positive macroH2A2

negative macroH2A2

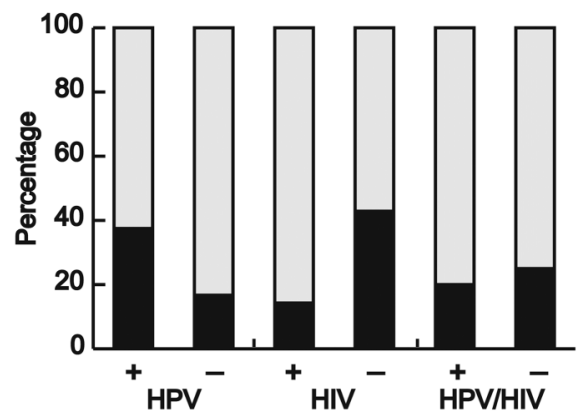

Figure 3 Summary of macroH2A2-positive samples segregated by human papilloma virus (HPV), HIV single and double infections among the anal squamous cell carcinoma cohort in this study.

potential of AIN II has remained equivocal for it contains a mixture of low-grade and high-grade histological features. ${ }^{20}$ In the literature, AIN II is mostly categorised into HSIL with AIN III, ${ }^{20} 212627$ while others grouped AIN II with AIN I for treatment and follow-up policies. ${ }^{27} 28$ In this study, we found that macroH2A2 expression was retained in all AIN I and II samples and lost in $38 \%$ of AIN III and the majority of anal SCC samples examined (figure 1B). This result suggests that macroH2A2 expression may be used as an independent molecular criterion for assessing the malignant potential of AIN lesions for treatment and follow-up policies. Furthermore, larger studies are needed to validate our findings and confirm that there is no loss in patients with AIN I/II.

As discussed above, the macroH2A2 histone variant is found in nucleosomes at promoters and in intergenic regions of the chromatin. ${ }^{13}$ Furthermore, macroH2A 2 is enriched in the inactivated $\mathrm{X}$-chromosome. ${ }^{8}$ The current data strongly suggest that macroH2A2 functions as an inhibitor of transcription and it has been shown to suppress the expression of E2F-regulated genes. ${ }^{102930}$ It is well established that the HPV E7 oncogene can inactivate the RB family of pocket proteins to activate E2F. ${ }^{31-33}$ In light of these facts, our finding that the majority of macroH2A2-positive anal SCC samples were also positive for HPV indicates that the inactivation of RB by E7 might be sufficient for activation of E2F-regulated genes despite the continued expression of macroH2A2. On the other hand, macroH2A2 loss in HPV-negative anal SCC samples may contribute to activation of E2F-regulated genes to promote tumour cell proliferation. In this regard, the preferential loss of macroH2A2 expression in HIV-positive SCC samples may be important to promote tumour development. Besides E2F activation, loss of macroH2A2 is likely to have a more global effect on gene expression promoting the development of anal SCC. The fact that macroH2A2 expression was also lost in the majority of AIN III suggests that inactivation of macoH2A2 is likely to be an early event in the malignant transformation of anal neoplasm.

There are a few limitations in our study. In situations where there is a low incidence of anal neoplasm, ${ }^{34} 35$ the number of patients is small and subject to be insufficient of statistical power in the difference of oncological outcome. On the other hand, this is a single-institution retrospective analysis with certain selection bias and divergent treatment. Further work would need to be done with larger cohorts in order to test the relationship between macroH2A2 expression and HPV or HIV status.

In conclusion, we present the association of histone variant macroH2A2 in the carcinogenesis of anal neoplasm. Positive
macroH2A2 predicts the better recurrence-free survival in patients with AIN. Thus, there is a potential for macroH2A2 expression to be a prognostic biomarker of anal neoplasm progression and an additional criterion in the categorisation and follow-up policies of AIN treatment.

\section{Take home messages}

- The macroH2A histone variants are encoded by two genes: $\mathrm{H} 2 \mathrm{AFY}$ for macroH2A1 and H2AFY2 for macroH2A2, and have tumour suppressor function in lung cancer and melanoma studies.

- Significant correlation was noted between the loss of macroH2A2 expression and the progression of anal neoplasm from AIN I/II to AIN III and anal squamous cell carcinoma (SCC) $(p=0.002)$.

- In patients with AIN, time to recurrence was significantly shorter in the macroH2A2-negative than the macroH2A2-positive group ( $p=0.017)$.

- Human papilloma virus (HPV)-positive SCC samples were more likely to express macroH2A2 and HIV-positive SCC samples were less likely to express macroH2A2.

\section{Author affiliations}

'Department of Surgery, University of California San Diego Health System, San Diego, California, USA

${ }^{2}$ Rebecca and John Moores Cancer Center, University of California San Diego Health System, San Diego, California, USA

${ }^{3}$ Department of Colorectal Surgery, Kaohsiung Chang Gung Memorial Hospital and Chang Gung University College of Medicine, Kaohsiung, Taiwan

${ }^{4}$ Department of Pathology, School of Medicine, University of California, San Diego, California, USA

${ }^{5}$ Department of General Surgery, Baystate Medical Center, Springfield, Massachusetts, USA

${ }^{6}$ Division of Hematology-Oncology, Department of Medicine, School of Medicine, University of California, San Diego, California, USA

${ }^{7}$ Department of Surgery, Veteran's Administration Medical Center, University of California, San Diego Healthcare System, San Diego, California, USA

\section{Handling editor Cheok Soon Lee}

Acknowledgements We thank Sridevi Priya for her valuable suggestion in correcting the manuscript.

Contributors W-HH collected data, performed statistic analysis and drafted the manuscript. KM evaluated and discriminated the slides. JCS supplied antibody and assisted immunohistochemistric stain. LL collected data and performed immunohistochemistric stain. JY-JW, BC and SR conceived the study and revised the manuscript. All authors approved the final manuscript.

\section{Competing interests None declared.}

Ethics approval The research was approved by the UCSD Human Protections Program (IRB). Under UCSD IRB approved, the retrospective study fit criteria for exemption; therefore, informed consent for the study was deemed unnecessary.

Provenance and peer review Not commissioned; externally peer reviewed.

Open Access This is an Open Access article distributed in accordance with the Creative Commons Attribution Non Commercial (CC BY-NC 4.0) license, which permits others to distribute, remix, adapt, build upon this work non-commercially, and license their derivative works on different terms, provided the original work is properly cited and the use is non-commercial. See: http://creativecommons.org/ licenses/by-nc/4.0/

\section{REFERENCES}

1 Monteiro FL, Baptista T, Amado F, et al. Expression and functionality of histone H2A variants in cancer. Oncotarget 2014;5:3428-43.

2 Pehrson JR, Fried VA. MacroH2A, a core histone containing a large nonhistone region. Science 1992;257:1398-400.

3 Chakravarthy S, Gundimella SK, Caron C, et al. Structural characterization of the histone variant macroH2A. Mol Cell Biol 2005;25:7616-24. 
4 Posavec M, Timinszky G, Buschbeck M. Macro domains as metabolite sensors on chromatin. Cell Mol Life Sci 2013;70:1509-24.

5 Karras Gl, Kustatscher G, Buhecha HR, et al. The macro domain is an ADP-ribose binding module. EMBO J 2005;24:1911-20.

6 Kustatscher G, Hothorn M, Pugieux C, et al. Splicing regulates NAD metabolite binding to histone macroH2A. Nat Struct Mol Biol 2005;12:624-5.

7 Costanzi C, Pehrson JR. MACROH2A2, a new member of the MARCOH2A core histone family. J Biol Chem 2001:276:21776-84.

8 Chadwick BP, Willard HF. Histone H2A variants and the inactive $X$ chromosome: identification of a second macroH2A variant. Hum Mol Genet 2001;10:1101-13.

9 Tanasijevic B, Rasmussen TP. X chromosome inactivation and differentiation occur readily in $\mathrm{ES}$ cells doubly-deficient for macroH2A1 and macroH2A2. PLoS One 2011;6:e21512.

10 Buschbeck M, Uribesalgo I, Wibowo I, et al. The histone variant macroH2A is an epigenetic regulator of key developmental genes. Nat Struct Mol Biol 2009;16:1074-9.

11 Gaspar-Maia A, Qadeer ZA, Hasson D, et al. MacroH2A histone variants act as a barrier upon reprogramming towards pluripotency. Nat Commun 2013;4:1565

12 Pehrson JR, Changolkar LN, Costanzi C, et al. Mice without macroH2A histone variants. Mol Cell Biol 2014;34:4523-33.

13 Yildirim O, Hung JH, Cedeno RJ, et al. A system for genome-wide histone variant dynamics in ES cells reveals dynamic macroH2A2 replacement at promoters. PLOS Genet 2014;10:e1004515.

14 Schnöder TM, Arreba-Tutusaus P, Griehl I, et al. EPO-induced erythroid maturation is dependent on Plcgamma1 signaling. Cell Death Differ 2015;22:974-85.

15 Rodríguez-Paredes M, Esteller M. Cancer epigenetics reaches mainstream oncology. Nat Med 2011:17:330-9.

16 Cantariño N, Douet J, Buschbeck M. MacroH2A-an epigenetic regulator of cancer Cancer Lett 2013:336:247-52.

17 Sporn JC, Kustatscher $\mathrm{G}$, Hothorn $\mathrm{T}$, et al. Histone macroH2A isoforms predict the risk of lung cancer recurrence. Oncogene 2009;28:3423-8.

18 Kapoor A, Goldberg MS, Cumberland LK, et al. The histone variant macroH2A suppresses melanoma progression through regulation of CDK8. Nature 2010:468:1105-9.
19 Siegel R, Ma J, Zou Z, et al. Cancer statistics, 2014. CA Cancer J Clin 2014:64:9-29.

20 Bejarano PA, Boutros M, Berho M. Anal squamous intraepithelial neoplasia. Gastroenterol Clin North Am 2013;42:893-912.

21 Zhang J, Martins CR, Fansler ZB, et al. DNA methylation in anal intraepithelial lesions and anal squamous cell carcinoma. Clin Cancer Res 2005;11:6544-9.

22 Uronis HE, Bendell JC. Anal cancer: an overview. Oncologist 2007;12:524-34.

23 Ahmed S, Eng C. Optimal treatment strategies for anal cancer. Curr Treat Options Oncol 2014;15:443-55.

24 Ramamoorthy S, Liu YT, Luo L, et al. Detection of multiple human papillomavirus genotypes in anal carcinoma. Infect Agent Cancer 2010;5:17.

25 Schiffman $\mathrm{M}$, Castle $\mathrm{PE}$, Jeronimo J, et al. Human papillomavirus and cervical cancer. Lancet 2007:370:890-907.

26 Smyczek $P$, Singh AE, Romanowski B. Anal intraepithelial neoplasia: review and recommendations for screening and management. Int J STD AIDS 2013;24:843-51.

27 Abbasakoor F, Boulos PB. Anal intraepithelial neoplasia. Br J Surg 2005:92:277-90

28 Scholefield JH. Anal intraepithelial neoplasia. Br J Surg 1999;86:1363-4.

29 Zhang R, Chen W, Adams PD. Molecular dissection of formation of senescence-associated heterochromatin foci. Mol Cell Biol 2007;27:2343-58.

30 Narita $\mathrm{M}$, Nũnez $\mathrm{S}$, Heard $\mathrm{E}$, et al. Rb-mediated heterochromatin formation and silencing of E2F target genes during cellular senescence. Cell 2003;113:703-16.

31 Helt AM, Galloway DA. Mechanisms by which DNA tumor virus oncoproteins target the Rb family of pocket proteins. Carcinogenesis 2003:24:159-69.

32 Chellappan SP, Hiebert S, Mudryj M, et al. The E2F transcription factor is a cellular target for the RB protein. Cell 1991;65:1053-61.

33 Yim EK, Park JS. The role of HPV E6 and E7 oncoproteins in HPV-associated cervical carcinogenesis. Cancer Res Treat 2005;37:319-24.

34 Meyer JE, Panico VJ, Marconato HM, et al. HIV positivity but not HPV/p16 status is associated with higher recurrence rate in anal cancer. J Gastrointest Cancer 2013;44:450-5.

35 Cotter $\mathrm{M}$, Kelly $\mathrm{M}, \mathrm{O}^{\prime}$ Connell $\mathrm{P}$, et al. Anal intraepithelial neoplasia: a single centre 19 year review. Colorectal Dis 2014;16:777-82. 